\title{
Leistungsträger als Kunden sehen
}

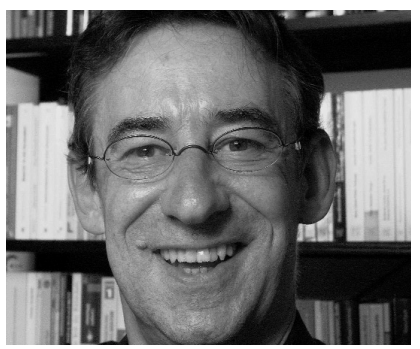

VON HARALD CHRISTA Prof. Dr. Harald Christa ist Hochschullehrer für Sozialmanagement an der Evangelischen Hochschule in Dresden. Zudem ist er Gesellschafter und Mitarbeiter der auf den sozialen Sektor spezialisierten Unternehmensberatung xit $\mathrm{GmbH}$ forschen.planen. beraten in Nürnberg, Dresden und Berlin. Seine hauptsächlichen Tätigkeitsfelder sind Marketingberatung, Netzwerkentwicklung und Evaluation. E-Mail harald.christa@ehs-dresden.de

\author{
Abnehmende finanzielle Potenzen öffentlicher Haus- \\ halte schlagen sich notgedrungen auf die Sozialmärkte \\ nieder. Selbst bei steigendem Bedarf müssen wir davon \\ ausgehen, dass die Budgets der öffentlichen Träger \\ bestenfalls stagnieren. In das Zentrum der Unter- \\ nehmensphilosophie ist deshalb stärker die Beziehung \\ zu den Kostenträgern rücken, weil sie die für den \\ Markterfolg entscheidenden Nachfrager sozialer \\ Dienstleistungen sind.
}

In Zeiten der finanziellen Krise öffentlicher Haushalte reagieren viele freie Träger fast instinktiv zunächst einmal mit einer Intensivierung ihrer Fundraisingaktivitäten. Jedoch stagnieren die verfügbaren Spenden-, Sponsoren und sonstigen Drittmittel seit Jahren, in einigen Regionen nehmen sie im Sog der wirtschaftlichen Entwicklung sogar ab. Das Bemühen um diese Mittel verspricht selbst dann wenig Erfolg, wenn die Basis eines erfolgreichen Fundraising, nämlich ein hervorragendes Image (Bekanntheit und Beliebtheit), bei den Spendern und den Sponsoren gegeben ist.

In erwerbswirtschaftlichen Märkten ist eine preisliche Reaktion als Folge sinkender Nachfragepotenziale zu erwarten, um den Anreiz für einen Kauf zu verstärken. Auch für Träger der Sozialen Arbeit ist eine Preissenkung eine gegebenenfalls auch kurzfristig zu erwägende Maßnahme, wenn der Kostenträger zu erkennen gibt, dass die Auftragsvergabe auf absehbare Zeit an preisliche Prioritäten gekoppelt ist. In der Praxis werden bereits von einigen Trägern Rabatte unter Berücksichtigung der Zeitpunkte der Inanspruchnahme als Anreiz für die öffentlichen Haushalt gegeben. Weitere Formen der Rabattgewährung bezie- hen sich auf Kundentreue und auf Mengen der Inanspruchnahme.

In leistungs- und kostenkalkulatorischer Hinsicht dominiert dann für den Anbieter allerdings das "Target Costing « (»Was darf eine Dienstleistung kosten, damit sie in Anspruch genommen wird? «).

Preispolitische Maßnahmen haben darüber hinaus den entscheidenden Nachteil, dass sie von Konkurrenten leicht kopiert werden können, entsprechende Wettbewerbsvorsprünge sind oft innerhalb kurzer Zeit eingeholt. Preispolitische Maßnahmen sind nicht angezeigt, wenn ein Träger bereits am unteren Ende der Kostenlinie anbietet und die Organisation auf geringe oder keine Rücklagen zurückgreifen kann. Lediglich finanzstarke Anbieter werden realistischerweise einen vom Preis dominierten Markt und eine hiermit korrespondierende Verdrängungsstrategie gegenüber finanzschwächeren Konkurrenten anstreben.

\section{Den Kostenträger als "Kunden« aufwerten}

Intensiviertes Fundraising und preisliche Reaktionen sind also nicht oder nur kurzfristig eine probate Reaktion 
auf abnehmende finanzielle Potenzen öffentlicher Haushalte.

Aus Sicht des Marketing ist eine dezidiertere Hinwendung zum Kostenträger als »Kunden « die sinnvollere Konsequenz für Anbieter solcher sozialen Dienstleistungen, deren finanzielle Grundlage überwiegend oder ganz von dieser Quelle abhängig ist. Die mikroökonomische Perspektive des Marketing legt nahe, in die organisationale Dispo- sition stärker als bislang die für den Markterfolg relevante »customer-based-view « zu integrieren und hieraus Wettbewerbsvorteile zu generieren. Jedoch darf dies nicht mit einer gleichzeitigen Abwendung von der Adressatenperspektive verbunden sein, vielmehr muss ein ausbalancierter Ansatz realisiert werden, der die Parameter des sozialwirtschaftlichen Leistungsdreiecks marktgerecht austariert.

\section{Literatur-Tipps zum Sozio-Marketing}

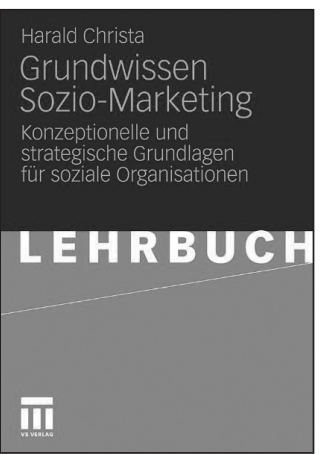

Aufgrund der Marktentwicklung in nahezu allen Bereichen der Sozialen Arbeit gewinnt Marketing, genauer: das Sozio-Marketing, als sozialwirtschaftliches Instrument an Bedeutung. Erfolgreiches Marketing bedarf einer fundierten konzeptionellen Grundlage, was häufig übersehen wird. Das neue Buch von Harald Christa bietet differenziertes Wissen zur Entwicklung wettbewerbsorientierter Marketing-Strategien. In einzelnen Schritten werden Begriffe und Konzepte zu Umfeldbeobachtungen, fundierten Marktanalysen und marktbezogenen strategischen Entscheidungen zur Kundenund Marktsegmentierung erklärt und aufgebaut. Vor allem wird klar vermittelt, dass die Faktoren »Information « und "Strategie« im Marketing von Institutionen des sozialen Sektors über den Erfolg maßgeblich mitentscheiden, und dass zukunftsfähiges Marketing mehr sein muss als PR und Werbung.

Harald Christa: Grundwissen Sozio-Marketing. Konzeptionelle und strategische Grundlagen für soziale Organisationen. VS-Verlag für Sozialwissenschaften, Wiesbaden 2010. 326 Seiten. 22,95 Euro. ISBN 978-3-531-17010-7.

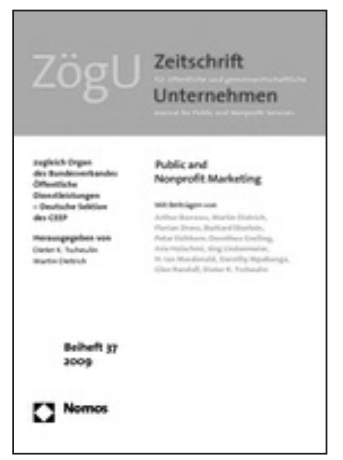

Marktorientierte Managementansätze gehören zu den relevanten Anforderungen, die inzwischen an ein Public und Non-Profit Management gestellt werden. Obwohl sich öffentliche und Non-Profit-Unternehmen intensiv mit einer anspruchsgruppenorientierten Sichtweise auseinandersetzen, hat die Übertragung von marktorientierten Managementansätzen im Rahmen eines Public und Non-Profit Marketings bisher nicht die notwendige Aufmerksamkeit erfahren. Das Beiheft 37 der Zeitschrift für öffentliche und gemeinwirtschaftliche Unternehmen widmet sich diesem Gebiet mit Aufsätzen zum Thema der Anwendung von Marketing und marktorientierten Managementansätzen im Public und Non-Profit Management. In diesem Heft werden unter anderem Aspekte und Methoden zur Bestimmung von Preisbereitschaften, Public-Private-Partnerships als besondere Form des marktorientierten Managements in öffentlichen Organisationen und weitere, auch aus dem internationalen Umfeld stammende Anwendungsgebiete dargestellt und erörtert. Das Beiheft leistet damit einen wesentlichen Beitrag zur Standortbestimmung eines marktorientierten Ansatzes im Public- und Non-Profit-Management nicht nur im deutschsprachigen Raum, sondern auch im internationalen Kontext.

Dieter K. Tscheulin, Martin Dietrich: Public and Nonprofit Marketing. Beiheft der Zeitschrift für öffentliche und gemeinwirtschaftliche Unternehmen. Beiheft 37/2009. Nomos Verlagsanstalt, Baden-Baden 2009. 144 Seiten. 38,- Euro. ISBN 978-3-8329-4925-9.
Die Marktstrategie eines Trägers kann dabei durchaus expansiv ausgelegt sein. Der Kern der Marketingstrategie als Leitfunktion organisationalen Agierens muss jedoch deutlich erkennbar auf »Social Return on Investment « unter Berücksichtigung der von den öffentlichen Finanziers artikulierten Bedarfslagen ausgelegt sein.

\section{Leistungspolitik überdenken}

Für Anbieter mit einem höheren Leistungspotenzial ist aus unserer Sicht auch in Zeiten finanzieller Krisen öffentlicher Haushalte die möglichst umgehende Hinwendung zur Strategie der "positionierten Qualität « durch »bedarfsgerechte Leistung « anzuraten. Diese Strategie erscheint im Übrigen auch für das soziale Selbstverständnis Freier Träger langfristig angemessener.

Dabei ist jedoch zu bedenken, dass »Qualität « von einem für Effizienz und Effektivität sensibilisierten Kostenträger nur nachgefragt werden kann, wenn der wahrgenommene Nutzen höher ist als die mit einer Leistung einhergehenden Kosten und Investitionen. Sollte eine basale Bereitschaft des öffentlichen Auftraggebers vorliegen, Qualität durch Inanspruchnahme zu honorieren, können über leistungs-, distributions- und kommunikationspolitische Maßnahmen Marktpositionen gestärkt und sogar ausgebaut werden.

Marketing korrespondiert in einem solchen Falle signifikant mit einem Qualitätsmanagement, welches sich auf Kundenwahrnehmung und Wirkungsaspekten konzentriert. Die übliche Kette des Qualitätsdenkens in der Sozialen Arbeit, wonach zunächst die Struktur-, dann die Prozess- und schließlich erst am Ende die Ergebnisqualität in die Betrachtungen einbezogen wird, muss sich umkehren zu einem Primat des Ergebnisses (Outcome und Impact, Social Return on Investment).

$\mathrm{Zu}$ nutzen sind die Potenziale, die für soziale Organisationen in einem verbesserten fachlichen Controlling und einer darauf ausgerichteten Informationspolitik gegenüber dem Kostenträger als » institutionellen Kunden « bestehen. In die fachliche Entwicklung müssten sie so direkt als möglich einbezogen werden, um eine Bindung im Sinne eines »Customer-Relationship-Managements « zu erzielen. Diese Strategie 
wird bereits von einigen Trägern der Jugendhilfe in erfolgreicher Weise im $\mathrm{Zu}$ ge von Hilfeplanungen realisiert.

Möglich sind jedoch auch leistungspolitische Maßnahmen, die über diese direkt am pädagogischen Kontext ansetzende Strategie hinausgehen. Eine die Kernleistung flankierende Servicepolitik sollte auf die umfassende und dem Kundenbedarf entsprechende Vermittlung von Vertrauen und die Erhöhung der Bindung an die Leistungspotenziale konzentriert werden.

Eine solche »Premium-Strategie « ist dann von besonderer Bedeutung, wenn Kostenträger bei der Auswahl von verschiedenen Anbietern sozialer Dienstleistungen noch nicht auf spezifische Indikatorensets für die Bestimmung der faktischen Leistungsstärke der sozialen Organisation zurückgreifen können. Dies gilt auch für die zuwendungsfinanzierten Ressorts. Relevant für die »Attraktivität « eines Angebots und die Einschätzung der Angemessenheit der Kosten ist für engagierte und fachlich kompetente Kostenträger und der Entscheider in Gremien wie dem Jugendhilfeausschuss zunehmend weniger die althergebrachte Lyrik der Konzeptionen, sondern der Fokus auf Nutzenstiftung und die Kohärenz mit dem festgestellten sozialräumlichen Bedarf.

Erfolg in der Akquise von Zuwendungen und anderen Fördermitteln ergibt sich dann über eine Korrelation zwischen dem Erwartungshorizont des Auftraggebers und dem (belegbaren und belegten) Leistungspotenzial des Anbieters.

\section{Marken schaffen}

$\mathrm{Zu}$ bedenken ist, dass lediglich über nachgewiesene Leistungspotenziale (al- so Wirkungen) und die Aufrechterhaltung dieser entscheidenden qualitativen Momente mittelfristig eine Markenpolitik auch im Sozialbereich erfolgreich implementiert werden kann.

In der Praxis wird die ausschlaggebende Bedeutung von Markierungen chen Grundlagen der Leistungserbringung einzugehen hat. Andererseits ist zu konstatieren, dass es kaum Alternativen gibt, wenn eine durchgehende Identifikation von Marke und Angebot bei den Kunden sowie eine klare Abgrenzung im Anbietergefüge ange-

\section{"Das übliche Denken in der Sozialen Arbeit -}

\section{zunächst Struktur-, dann Prozess- und \\ schließlich Ergebnisqualität - \\ muss umgedreht werden"}

nicht immer hinreichend berücksichtigt: Über Marken können »Informationsbeschaffungskosten « der Kunden beim Einkauf verringert (bis im Idealfall minimiert) werden. Notwendige Bedingung hierfür ist jedoch, dass sich für die Kostenträger als Nachfrager ein ebenso klarer wie nachhaltiger Unterschied zwischen den Angeboten und den damit korrespondierenden Marken ergibt. Lediglich auf diese Weise können Präferenzen entstehen und Kundenbindungen über Marken dauerhaft realisiert werden.

$\mathrm{Zu}$ erkennen ist allerdings auch, dass mit einer Marke ein positioniertspezifisches Leistungsversprechen und damit eine Reihe von Verpflichtungen mit gegebenenfalls hohem Aufwand für die soziale Organisation verbunden sind. Das Problem der Gewährleistung von verlässlicher Qualität verweist auf die enge Verbindung, die bei der Markenpolitik das Marketing mit dem Management von Leistungsfähigkeit sowie vielen fachli- strebt werden soll. Kaum anders als durch die Koppelung von Marke und dauerhaft erwartbarer Qualität ist eine nachhaltige Präferenz eines Kunden zu erreichen.

\section{Neue Kundengruppen erschließen}

In distributionspolitischer Hinsicht ist die Erschließung neuer Kunden wie Selbstzahler und Kostenträger aus anderen Regionen anzuraten. Mitunter können damit auch gefährliche Abhängigkeiten von einem öffentlichen Haushalt als Monopolkunden reduziert werden. Zweifellos spielen in akquisitorischer Hinsicht die Potenziale der Verbesserung auch von persönlichen Kundenbeziehungen eine wichtige Rolle, schließlich dürfen wir in den kommenden Jahren nicht durchgängig von Entscheidungen öffentlicher Träger bei der Wahl eines Anbieters ausgehen, welche ausschließlich auf objektiven Indikatoren basieren.

\section{Was versteht man eigentlich unter Social Return on Investment?}

Social Return on Investment (SROI; deutsch: "Soziale Investitionsrechnung ") ist ein neuerer Ansatz, der sich mit der Bewertung des durch (soziale) Projekte geschaffenen gesellschaftlichen Mehrwerts beschäftigt. Der Entwicklung des SROI-Ansatzes im angelsächsischen Raum ging dabei die Überlegung voraus, wie die Auswirkungen, die durch die Arbeit insbesondere sozialer Organisationen und Projekte im Sinne des Gemeinwohls entstehen, gemessen und finanziell bewertet werden können. Der SROI ist dabei eine Methode, die die klassischen finanziellen Bewertungsmethoden (ROI) um einen sozialökonomischen und umweltpolitischen Wert erweitert und damit auch für Non-Profit-Organisationen und -Unternehmen anwendbar macht.

Quelle: http://de.wikipedia.org/wiki/Social_Return_on_Investment 


\section{Kommunikationspolitik optimieren}

Mit der Pflege personaler Beziehungen kann auch eine dezidierte Convenience-Politik verbunden werden, die sich auf die Frage konzentriert, welche Servicepotenziale (informationeller und anderer Natur) noch nicht ausgereizt sind.

Die Servicepolitik geht in einzelnen Feldern des Sozialen auch bis hin zur physischen Distribution als Akquisitionsstütze, wenn Hol- und Bringdienste beispielsweise in den Sektoren der Kindertagesstätten und der Hilfen für Menschen mit Behinderung optimiert werden, um der Klientel eine Inanspruchnahme zu erleichtern.

Selbstverständlich zählen zu den modernen distributionspolitischen Maßnahmen mittlerweile auch Optimierungen in der Kontakt- und Geschäftsanbahnung via Internet. Nicht wenige Kostenträger informieren sich inzwischen auf diesem Wege über die konzeptionell signalisierten Leitungspotenziale der sozialen Organisationen.

Sozialen Organisationen stehen eine Fülle kommunikationspolitischer Maßnahmen zur Verfügung, die der Imagepflege in der Bevölkerung und der Werbung von Spenden ebenso dienen können wie dem sozialpolitischen Lobbyismus.

Aus Sicht eines an tragbarer Qualität und mittelfristigem Überleben ausgerichteten Marketing ist die Ausrichtung der Unternehmenskommunikation an die Bedarfe der Kostenträger als institutionelle Schlüsselkunden allerdings mindestens ebenso wesentlich. Der kommunikative Fokus ist dabei eindeutig auf Qualität durch bedarfsgerechte Angebote sowie dem Nachweise von Wirkung zu legen.

\section{Aktuelle}

\section{Neuerscheinung}

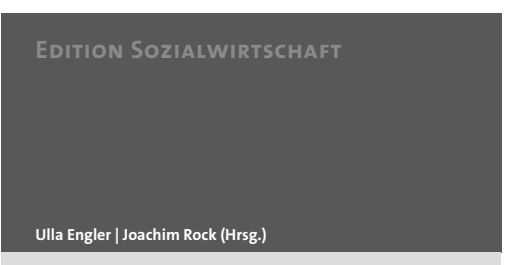

\section{Die Zukunft des \\ Sozialen - in Europa?}

Soziale Dienste und die europäische Herausforderung

C Nomos

\author{
Die Zukunft des \\ Sozialen - in Europa? \\ Soziale Dienste und die \\ europäische Herausforderung \\ Herausgegeben von RAin Dr. \\ Ulla Engler und Dr. Joachim Rock \\ 2011, ca. 100 S., brosch., ca. 19,- $€$ \\ ISBN 978-3-8329-5689-9 \\ (Edition Sozialwirtschaft) \\ Erscheint ca. März 2011
}

Der Einfluss des europäischen Rechts auf die nationale Sozialpolitik ist in den vergangenen Jahren erheblich gewachsen. Von besonderer Bedeutung ist dabei der Einfluss des europäischen Wettbewerbs- und Vergaberechts auf die Gesundheits- und Sozialpolitik in Deutschland. Dies betrifft die Sozialwirtschaft in besonderer Weise. Gleichzeitig wächst die Bedeutung des Sozialen im Gemeinschaftsrecht, beispielsweise durch die Charta der Grundrechte der EU. Vor diesem Hintergrund stellt sich die Fragen nach dem wechselseitigen Einfluss der Rechtsbereiche und den materiellen und formellen Gestaltungsmöglichkeiten der Sozialpolitik in Deutschland ebenso wie nach dem Verhältnis von Solidarität und Subsidiarität.

Das Werk beinhaltet aktuelle Beiträge einer wissenschaftlichen Tagung sowie weitergehende Artikel zu Hintergründen, einzelnen Themenbereichen und rechtlichen und politischen Konsequenzen. Die Autoren sind namhafte Experten aus Rechtswissenschaft, Politikwissenschaft und Praxis.

Der Band richtet sich an Beschäftigte aus Wirtschaft und Verwaltung sowie an Wissenschaftler und Interessierte, die sich vertieft mit den Folgen des Europäisierungsprozesses für die Sozial- und Gesundheitswirtschaft in Deutschland befassen möchten.

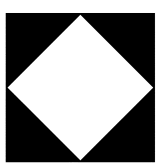

\section{Nomos}

Bitte bestellen Sie im Buchhandel oder versandkostenfrei unter $>$ www.nomos-shop.de 